Philologische Praxis I 
Alfred Behrmann

\section{PHILOLOGISCHE PRAXIS I}

Erfahrungen und Reflexionen

J.B. Metzlersche Verlagsbuchhandlung

StUTTGART 


\section{CIP-Titelaufnahme der Deutschen Bibliothek}

Behrmann, Alfred:

Philologische Praxis: Erfahrungen und Reflexionen / Alfred Behrmann.

- Stuttgart: Metzler

1 (1991)

ISBN 978-3-476-00724-7

ISBN 978-3-476-00724-7

ISBN 978-3-476-03334-5(eBook)

DOI $10.1007 / 978-3-476-03334-5$

Dieses Werk einschließlich aller seiner Teile ist urheberrechtlich geschützt. Jede Verwertung außerhalb der engen Grenzen des Urheberrechtsgesetzes ist ohne Zustimmung des Verlages unzulässig und strafbar. Das gilt insbesondere für Vervielfältigungen, Übersetzungen, Mikroverfilmungen und die Einspeicherung und Verarbeitung in elektronischen Systemen.

(C) 1991 Springer-Verlag GmbH Deutschland

Ursprünglich erschienen bei J.B. Metzlersche Verlagsbuchhandlung und Carl Ernst Poeschel Verlag GmbH in Stuttgart 1991 


\author{
FOR \\ Arthur Hyman \\ The Man And The Scholar \\ With Admiration
}

Daß man nicht überall mit uns zufrieden sein sollte, war ja die Absicht...

Goethe an Schiller, 26.10.1796 


\section{INHALT}

\section{Vorrede \\ 1}

Tractatus paedagogico-philologicus

6

Philologie: der Gelehrte und sein Vorhaben

19

Literarische Studien oder Verteidigung eines gemäßigten Empirismus

35

Vom möglichen Nutzen des Philologen 55

Denkwürdigkeiten aus der jüngeren Geschichte eines germanistischen Instituts (1969-84)

70

Eine akademische Dichterlesung

100

Literarische Erziehung für Lehrer und andere

105

Anmerkungen

120

Drucknachweise

129 


\section{VORREDE}

Das Tun des Philologen besteht im Lesen und Schreiben, im Reden und Vortragen; er gibt heraus, übersetzt, kommentiert und interpretiert; er lehrt. Seine Aufgabe ist die Darstellung der Literatur. Soll von diesem Beruf die Rede sein, wäre als erstes eine Erörterung solcher und ähnlicher Tätigkeiten zu erwarten, die ihn ausmachen. Davon soll ein anderes Buch im einzelnen handeln. Hier erscheint der Rahmen, in dem der Philologe arbeitet. Dies aus gutem Grund: weil es >den< Philologen natürlich nicht gibt. Es gibt Philologen, und deren Ansichten von ihrem Beruf unterscheiden sich, seis, weil sie verschieden spezialisiert, seis, weil sie verschieden geartet sind. Was der einzelne tut, wird von Umständen, die heute Rahmenbedingungen heißen, wenn nicht geprägt, so doch mit bestimmt. Auch wer sich auflehnt dagegen, weil es ihn bedrückt, bleibt darauf gerichtet: indem er nicht tut, was die Umstände nahelegen, wird sein Tun zum Einspruch.

Hutten, der Humanist und Philologe, empfand es als Lust, in seiner Zeit zu leben, weil Wissenschaften und Künste aufblühten. Im 20. Jahrhundert hat sich eine Stimme wie seine nicht erhoben. Umgekehrt haben manche, die sich Hutten im Geist verbunden fühlten, erklärt, ihr Zeitalter versinke in Unwissenheit und Barbarei. Es verspricht also Aufschluß, die Umstände zu betrachten, unter denen ein Philologe sein Gewerbe treibt, die Anstöße und Hemmungen, die von den Umständen auf ihn ausgehn, die Art, wie er mit seiner Arbeit darauf reagiert.

Nun wird ein Gelehrter, zumal ein deutscher, einen Gegenstand, der ihn ernsthaft beschäftigt, nicht anders aufgreifen als in Form einer Geschichte und Systematik, zumindest einer Theorie. Ein Thema wie die Praxis des Philologen in kurzen Aufsätzen zu behandeln scheint kaum seriös, deutet auf beiläufiges, vielleicht vorübergehendes Interesse. Liefern die Aufsätze zudem kein 
Bild, das auf genauen, umfassenden und repräsentativen Erhebungen beruht, ist dem Verdacht des Unverbindlichen schwer zu entgehn.

Das Meßbare verwaltet die Sparte Bildungsökonometrie des Instituts für Bildungsforschung in der Max-Planck-Gesellschaft. Dort wird die Ausbildungskapazität, der input, output und dropout erhoben, die Erfolgsquote in Gestalt von Examensprädikaten registriert. Man wird aber annehmen dürfen, daß auch Bildungsforscher zögern, aus solchen Daten Schlüsse auf vermittelte und erworbene >Bildung zu ziehn. Dabei ist Bildung ein Phänomen, das am ehesten im Umkreis der Künste, unter den Wissenschaften also bei denen zu suchen ist, die sich, wie die Philologie, mit Kunst befassen.

Die Behauptung, Philologie befasse sich mit Kunst, ist zwar schwer zu widerlegen, da sie unter anderm mit Dichtung zu tun hat, die noch immer bei den Künsten ressortiert. Doch dürfte die Ansicht, der Philologe sei ein Kunstwissenschaftler wie der Kunsthistoriker und der Musikwissenschaftler, bei der Mehrheit der Zunft auf wenig Beifall, z.T. auf entrüstete Ablehnung stoBen. Denjenigen, die ihr Erscheinungsbild prägen, ist die Rede von Kunst verdächtig; es sei denn, sie fühlten sich von Derrida zu eigner Kunstproduktion vermittels philosophischen Theoretisierens über Literatur ermuntert. Ihr Verdacht erhebt sich, sobald das Wort mit einem Zungenschlag an ihr Ohr dringt, der ihr eigener sein müßte, um nicht als falsch empfunden $\mathrm{zu}$ werden: niemand sonst bringt es beiläufig und unscheinbar genug heraus.

Das Erkenntnisinteresse gilt anderem als dem Kunstcharakter von Literatur. Ihn zu untersuchen, in dieser Untersuchung ein wichtiges Geschäft zu sehn rückt den dazu Gewillten an den Rand der Zunft. Nun ist Randständigkeit etwas, was Aufmerksamkeit, ja Sympathie heute eher erregt als ausschließt. Auch läßt sich vom Zentrum nur sagen, daß es ständig wechselt. Nach dem Krieg war es die Werkinterpretation, dann der Strukturalismus, der Marxismus, die Psychoanalyse, der Poststrukturalismus, der sich aufsträhnt in ein Mit-, Neben-, Über- und Ineinander von >Paradigmen`, deren Durchlaufgeschwindigkeit immer atem- 
beraubender, deren Muster immer kaleidoskopischer wird. So ist der Rand ein Ort, der sich zusehends bevölkert; denn die Kondition, die fortgesetzter Aufenthalt im Zentrum erfordert, grenzt an Wunder von Fixigkeit, Anpassungsvirtuosität und Streßresistenz.

Das Dasein am Rand hat viele Vorzüge, so die der Obskurität und des Zweifels am eigenen Treiben, der im Zentrum nicht auftritt, weil niemand Zeit findet, ihn vorzulassen. Die Randexistenz bedenkt also, wie sich die Schande erklärt, ein Leben lang nichts zu leisten, was die Zustimmung der jeweils Hervorragenden fände. Sie fragt sich, ob sie der Schauspieler ist, der ständig sein Stichwort verpaßt, ein ewig Verspäteter. Sie müßte, wenn dem so wäre, auf einer Bühne reüssiert haben, die nur eben schon abgeräumt ist. Sie versetzt sich zurück und stellt fest, daß sie im Dekor des Positivismus, der Geistesgeschichte, des Geworfenseins, der Zweiten Aufklärung so deplaziert gewirkt hätte wie in den Erregungen, die seither die Szene beherrschen. Hätte sie nur halbwegs das Zeug, an der Rampe zu figurieren, so käme sie aus einem Anlaß wie diesem als behender lazzista daher. Um dem Verdacht zu entgehn, hier belle jemand den Mond an, müßte sie mit einer Reflexionsakrobatik aufwarten, die an jene der Matadore heranreicht.

Dazu ist die Randexistenz, die hier spricht, weder aufgelegt noch imstande. Es geht ihr wie Eliot, der erklärte, für den »flight of abstruse reasoning « sei sein Geist zu schwer und konkret. [1] Sie wird verfahren, wie sie auch sonst verfährt, und holt sich Bestärkung dafür bei Paul Valéry. Der erklärte bei einem weit verwickelteren Thema als dem gegenwärtigen, nämlich einer bestimmten Theorie der Dichtkunst, sein fester Vorsatz sei, "nichts zu sagen, was nicht reine Feststellung von Tatsachen wäre, die jedermann in sich selbst oder von sich selbst aus beobachten oder mindestens durch eine einfache Überlegung bestätigt finden könnte.« [2]

Philologische Praxis stellt sich in zweierlei Form dar, als Ausübung durch die Approbierten und als Ausbildung der Adepten. 
Was im folgenden darüber gesagt wird, rührt her aus kontinuierlicher fünfundzwanzigjähriger Tätigkeit an einem Institut (dem germanistischen) und einer Hochschule (der Freien Universität), deren Bild unlösbar mit dem Phänomen der Studentenbewegung und der durch sie bewirkten Universitätsreform verbunden ist. Wie bekannt, trat die Bewegung hier früher, heftiger und folgenreicher auf als anderswo; sie prägt noch immer die äußre und innere Verfassung bestimmter Fächer.

Begreiflicherweise spielen Ort, Zeit und Umstände für die Praxis eine größere Rolle als für die abgehobnere Theorie. Freilich war gerade in Berlin die Praxis ein Moment, das nach Auffassung vieler die Theorie so entschieden verpflichtete, wie es ihr verpflichtet war. Beide, Theorie und Praxis, erschienen als Werkzeug zur Umbildung, einer möglichst vollständigen Umbildung, der Philologie, der Wissenschaft, ja der Gesellschaft. Dem Mitlebenden ergibt sich daraus ein Bild, das anderswo vermutlich ein andres geworden wäre. Gleichwohl ist ihm Belang, ja Repräsentanz nicht abzusprechen: es zeigt in besondrer Schärfe, was sich selbst als Maßstab empfand und weithin, wenn auch in Abstufungen, als maßgebend empfunden wurde. Das Präteritum raubt der Sache nur scheinbar ihre Aktualität. Nicht bloß, daß sich im gegenwärtigen Zustand das Anhalten ihrer Wirkung bezeugt, auch wenn das Spektakuläre dem Unscheinbaren gewichen ist: es zeigt sich, daß die Philologie - die Deutsche nicht fähig ist, genügend Substanz zu bilden, um sie selbst zu sein. Welche Turbulenz sie morgen beutelt, weiß niemand; umso näher liegt es, die von gestern zu bedenken: sie ist und bleibt, als Beispiel, symptomatisch.

Nicht jeder wird die Sache des Aufhebens wert finden, das hier davon gemacht wird. Daß auf dem Markt und an den Toren Geschrei herrscht, mag den Arbeiter im Weinberg der Philologie nicht stören. Er blickt auf den stetigen Gang seines Geschäfts mit der Ruhe dessen, der weiß, daß geduldige Arbeit am weitesten führt, das Unscheinbare seinen Lohn in sich selbst trägt 
und, wenn es gediegen ist, auch Schätzung findet, und zwar gerechte, nämlich die der Besonnenen.

Solch Quietismus mag beglückend sein. Es bedarf eines stillen Winkels und ergebner Seele dazu. Wem der Weltgeist in Gestalt sehr robuster und lärmender Agenten auf den Leib rückt, wen die Art seiner Arbeit zum Subjekt macht, vor dem gewarnt, ja das in den Anklagezustand versetzt wird, dem fehlt zur Ergebung der stille Sinn. Nicht, daß Gelassenheit ihm unerreichbar schiene oder nicht wünschenswert. Im Gegenteil. Nur stellt sie sich anders her als jene der Quietisten. Er genießt sie als Randständiger, der diesen Stand nicht gewählt hat, ihn aber heiterer einnimmt, nachdem er ein Vierteljahrhundert nolens volens den Markt und die Tore aus nächster Nähe erlebt hat, wo die Ansehnlichkeit ihre Spiele veranstaltet.

Ich danke Herrn Dr. Frank Heibert für Hilfe beim Vorbereiten des Manuskripts und insbesondere Herrn Dr. Fritz Minde für aufmerksame Teilnahme und kritischen Rat, bei dieser Arbeit wie bei mancher anderen. 